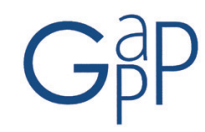

GESTIÓN Y ANÁLISIS DE POLÍTICAS PÚBLICAS, Nueva Época, nº 14 julio-diciembre 2015 ISSN: 1989-8991

DOI: http://dx.doi.org/10.24965/gapp.voi14.10292

\title{
Análisis de la aplicación de políticas públicas en el sector turismo. El caso de Colombia
}

\author{
Oscar Díaz Olariaga \\ Universidad Antonio Nariño (Colombia) \\ diaz_olariaga@uan.edu.co
}

\begin{abstract}
Resumen
Este trabajo presenta, explica y analiza como la aplicación y desarrollo de las políticas públicas y sectoriales impactaron en la evolución y transformación de la industria turística colombiana. Mediante un desarrollo cronológico se va presentando la aplicación de las diferentes regulaciones y políticas públicas y sus impactos socio-económicos en el turismo. Finalmente el trabajo se cierra describiendo el estado de situación actual del turismo colombiano, y mencionando sus grandes retos y desafíos a futuro.
\end{abstract}

Palabras clave

Colombia, turismo, políticas públicas, políticas turísticas

\section{Analysis of implementation of public policies in the tourism sector. The case of Colombia}

Abstract

This essay presents, explains and analyses the impact of the development and application of both public and sectoral policies which have revolutionized and transformed the Colombian tourist industry. The application of different regulations and public policy and the socio-economic impact of tourism is presented chronologically. Finally, this essay closes describing the current situation and discussing future challenges facing the industry in Colombia.

\section{Keywords}

Colombia, tourism, public policies, tourism policies. 


\section{INTRODUCCIÓN}

Este trabajo analiza como la aplicación y desarrollo de las políticas públicas consiguieron transformar a la industria turística colombiana, prácticamente inexistente, desde el punto de vista institucional, hace sólo tres décadas, hoy en día el sector de mayor generación de empleo del país y el tercer exportador por divisas y con una presencia creciente en el mapa turístico mundial.

La existencia de regulación y políticas públicas ha conseguido que el turismo colombiano esté experimentando un proceso de crecimiento imparable desde principios de la década de los 90 . El mercado interno ha reaccionado vigorosamente a las políticas aplicadas en el sector, las cuales han facilitado el control de ciertas variables críticas (seguridad, inversión, incentivos). Los colombianos volvieron a desplazase por las distintas regiones del país con fines de descanso y ocio, hecho favorable que está registrado en los indicadores sectoriales. La recuperación de la confianza en las instituciones, la reactivación del crecimiento económico, la percepción cada vez más positiva del país en el exterior, el aprovechamiento, por parte de los empresarios, de las ventajas creadas a través de los incentivos tributarios y la recuperación de la inversión extranjera directa son hechos que marcan la confluencia de una serie de factores que, capitalizados de manera eficiente, están llevando al turismo colombiano a ocupar un lugar privilegiado en la economía como un sector significativamente contribuyente para el logro de las metas económicas y sociales del país.

Como se verá en los datos presentados y analizados en este trabajo, el sector turístico colombiano pasa actualmente por un excelente momento. Se han canalizado esfuerzos públicos y privados para generar espacios de desarrollo para las iniciativas turísticas. Las políticas públicas emanadas de los diferentes planes y políticas sectoriales han contribuido progresivamente a afianzar el compromiso de los diversos entes del gobierno para apoyar al sector. Paralelamente los últimos planes nacionales de desarrollo ven ya al turismo como una industria relevante del país y definen grandes estrategias a seguir por el sector. Por otro lado, el turismo receptivo ha reaccionado favorablemente y empieza a mostrar un importante crecimiento del número de visitantes extranjeros que ingresan a Colombia.

Las autoridades públicas turísticas entienden que la favorabilidad del escenario actual implica enfrentar grandes retos. Superados los escollos más grandes para el mercado interno, que aún debe seguir creciendo y consolidándose, se asume el compromiso de captar corrientes internacionales significativas de turistas extranjeros. Para lograr este objetivo las autoridades saben que se debe priorizar el esfuerzo interinstitucional para fortalecer y posicionar productos de clase mundial en los cuales el país sea realmente competitivo. Como se verá en el análisis desarrollado, conseguidos ya indicadores de impacto, las autoridades turísticas se enfocan ahora en alcanzar estándares internacionales de competitividad y calidad, asignaturas pendientes en el desarrollo actual de la industria turística colombiana.

\section{MARCO TEÓRICO Y CONCEPTUAL}

\subsection{Análisis de políticas públicas}

El concepto de política pública denota un conjunto de principios, iniciativas, procesos y medios diseñados e implementados por el gobierno con el propósito de coordinar, regular y orientar la conducta de los agentes económicos y sociales hacia la consecución de objetivos específicos en uno o más sectores de interés nacional, regional y estatal. En otro orden, las políticas públicas son un modo de acción sólo concebible razonablemente dentro del marco de un sistema político, en el que el Estado tenga la facultad de regular, intervenir, impedir, compensar o reajustar los efectos indeseables de la lógica del mercado y de sus propias acciones (Velásquez, 2009). Y la noción misma de políticas públicas sólo tiene sentido dentro de marcos conceptuales que permitan ver el proceso de toma de decisiones públicas como el objeto específico de la ciencia política (Álvarez Díaz, 1992). Según Roth Deubel (2002) una política pública designa la existencia de un conjunto conformado por uno o varios objetivos colectivos considerados necesarios o deseables y por medios y acciones que son tratados, por lo menos parcialmente, por una institución u organización gubernamental con la finalidad de orientar el comportamiento de actores individuales o colectivos para modificar una situación percibida como insatisfactoria o problemática. Igualmente plantea cómo el análisis de las políticas públicas consiste en examinar una serie de objetivos, de medios y de acciones definidos por el Estado para transformar total o parcialmente la sociedad, por lo tanto, requiere una formulación, implementación y evaluación. Para Aguilar (1996) el análisis de política pública, es la evaluación de la cual, entre las varias opciones de política o decisión gubernamental, es la mejor decisión, o política, a la luz de determinados fines, restricciones y condiciones. Guerrero (1995) afirma que el análisis y evaluación de políticas públicas no se limita a describir simplemente los sucesos ligados a la aplicación de una política determinada, también pretende explicar por qué tuvo el efecto logrado y examinar si el problema que originó la actuación gubernamental fue finalmente resuelto 
y si se alcanzaron sus objetivos. Finalmente, Meny y Thoenig (1992) sugieren que el análisis de las políticas públicas constituye otra forma de aproximarse al problema del poder. Toda sociedad política está organizada a través de procedimientos, de jerarquías y de instituciones más o menos formales. El análisis de las políticas públicas invita, también, a romper las fronteras tradicionales de las disciplinas, tal como se han podido desarrollar a través de la profesionalización y la especialización del campo científico relacionado.

\subsection{Políticas públicas en turismo}

Los estudios sobre las políticas de turismo han sido un reto debido a ciertas dificultades metodológicas y también porque el turismo no siempre ha sido considerado como un campo académico estrictamente formal (Whitford, 2005; Hall y Jenkins, 1995; Hall, 1994). Sin embargo algunos autores (Scott, 2011) destacan que la política turística es un área relevante para ser estudiada debido a su importancia tanto práctica como teórica. Las investigaciones de políticas públicas de turismo ayudan a entender la conformación de las decisiones políticas y sus impactos en el desarrollo del turismo (Hall, 2008; Hall, Jenkins y Kearsley, 1997; Berno, 1996). Por lo tanto, existe la necesidad de una mayor atención a este tipo de estudios en la literatura (Torres, 2004; Matthews y Richter, 1991; Hall, 1989; Richter, 1983).

Bayón (1999) define la política turística como el conjunto de actuaciones y medidas emprendidas y realizadas por las administraciones públicas relativas a la actividad turística. Posteriormente, Monfort (2000) afirma que el establecimiento de una política turística conlleva, en definitiva, la articulación de unas estrategias, canalizadas por medio de planes, programas y medidas legislativas, que facilitan el alcance de los objetivos o metas predeterminados. Por su parte, Velasco (2004) indica que política turística es el conjunto, articulado y coherente, de decisiones y actuaciones que llevan a cabo los gobiernos en el ámbito territorial de su competencia, con la intención de alcanzar unos objetivos determinados, en relación con los hechos de distinta naturaleza que genera el proceso de atracción, estancia o residencia ocasional de ciudadanos; y además afirma que únicamente se puede hablar de política turística cuando las acciones que se van llevar a cabo, como objeto de ésta, repercuten directamente en el sector turístico. Posteriormente Velasco (2011) afirma que utilizando el análisis de políticas como perspectiva de análisis es posible proponer una definición de política turística operativa, enumerar cuáles son los elementos constitutivos de este tipo de acción pública y describir los instrumentos que pueden ser combinados por los decisores públicos para que, a través de la política diseñada, se alcancen los fines propuestos. Fayos-Solá (2004) afirma que la política turística es una técnica de gestión del conocimiento para investigar, difundir e implementar las reglas del juego a nivel macro. Más recientemente Rodríguez y Martínez (2009) definen política turística como el conjunto de acciones que impulsan actores públicos, en ocasiones en colaboración con otros actores no públicos, con la intención de alcanzar objetivos diversos relacionados con la variedad de fenómenos y relaciones que supone el proceso de atracción, estancia o residencia ocasional de ciudadanos en un territorio determinado. Y finalmente, Izard (2010) señala que la política turística, no es más que una especialización, o micro-política, que la administración pública usa para establecer una serie de directrices de ordenación, planificación, promoción y regulación de la actividad turística. En definitiva, la política turística comprende acciones, actuaciones, medidas, decisiones e, incluso, se habla de directrices realizadas por la administración pública bajo unos objetivos que responden, en términos generales, a la cualificación de la oferta y la atracción de la demanda.

\section{REVISIÓN DE LA LITERATURA A NIVEL COLOMBIA}

La literatura científica en materia exclusiva de análisis de políticas públicas turísticas en Colombia es muy escasa (Zúñiga y Castillo, 2012; Zúñiga et al., 2012), argumento que ha motivado la realización del presente trabajo de investigación. En la última década se pueden destacar los siguientes trabajos relacionados. Toro (2003) realiza un análisis descriptivo sobre los orígenes de la política nacional de turismo y luego de las políticas sectoriales turísticas en la década de los 90. Quintero (2009) realiza una investigación enfocada en la política turística y en los procesos de planificación y gestión de la actividad en Colombia, y su impacto o implicancia en el desarrollo y competitividad del turismo colombiano. La principal conclusión de su investigación es que contrariamente a la percepción (por entonces, año 2009) de que los factores de seguridad y mala imagen de Colombia son el único factor determinante del papel marginal del país en el mercado turístico internacional, también influye, y de forma determinante, la escasa importancia del turismo dentro de la política nacional, la debilidad institucional y la incoherencia de la política turística. Peña (2009) realiza un análisis de la política turística y el marco legal que la sustenta en cuatro países latinoamericanos (Colombia, Perú, México y Honduras). En base a las carencias y problemas detectados, el estudio sugiere (para los cuatro países analizados) que las políticas de turismo que se diseñen deben estar basadas en instrumentos legales sólidos, coherentes, transparentes, claros, equilibrados, fiables y consensuados, que les permitan orientar sus acciones hacia los objetivos prioritarios del desarrollo económico, social y ambiental sostenible de sus países, dejando atrás los enfoques tradicionalistas que se basaban sólo en tímidas y aisladas 
políticas sectoriales. Mediante un estudio desde el punto de vista de la competitividad Benavides y Venegas (2013) encuentran que el enfoque de la política turística colombiana no impacta realmente en la competitividad ya que no integra de forma eficiente los actores del clúster turístico. Restrepo (2014) presenta los avances alcanzados en el sector turismo en Colombia, durante el periodo 2004-2014, a través de un análisis cualitativo de las políticas públicas en la normatividad, planes de desarrollo sectorial y acciones específicas en gestión. Y finalmente, Benavides (2015) analiza los elementos clave de la política turística trabajada por Colombia durante la última década (2004-2014); el análisis se centra en los instrumentos de la política turística, particularmente en lo que se refiere al servicio turístico receptivo colombiano, explorando su impacto en la competitividad y el dinamismo del sector a futuro.

\section{METODOLOGÍA}

Eldesarrollo metodológico de estetrabajo deinvestigación, de carácter descriptivo, presenta cronológicamente la aplicación de las diferentes políticas públicas turísticas desde sus orígenes (incluso se inicia con el desarrollo histórico institucional del turismo en Colombia), y analiza sus resultados parciales y su influencia e impacto en la economía a través de la valoración y evaluación cuantitativa de un conjunto de indicadores relacionados, típicos de la industria turística. Al final, y ya en la actualidad, se presenta y detalla el estado de situación presente de los grandes indicadores y su impacto socio-económico, las evaluaciones y valoraciones generales del desarrollo de la industria, y finalmente se mencionan los objetivos y retos por conseguir.

\section{ORIGEN Y DESARROLLO INSTITUCIONAL DEL SECTOR TURISMO}

En Colombia la industria del turismo nace con el desarrollo los hoteles. Es recién en la década de los 20 y 30 del siglo Xx cuando se construyen e inauguran los primeros hoteles en algunas ciudades de Colombia. En sólo tres décadas, entre 1927 y 1957, se construyeron 23 hoteles de tamaño e importancia, distribuidos en las principales ciudades, incluidas las ciudades más turísticas en la costa del Caribe. Luego, en los 15-20 años posteriores, el desarrollo de hoteles cubriría las más importantes plazas del país (Moreno, 1981).

Ante el crecimiento del número de hoteles en el país, el gobierno ve la necesidad de crear un organismo para su reglamentación, por lo que mediante la Ley 86 del 26 de junio de 1931 creó el Servicio Central de Turismo con sede en Bogotá. Este organismo dependió, en ese entonces, del Ministerio de Industria y Comercio. Posteriormente, en 1957, el gobierno nacional crea la Empresa Colombiana de Turismo (mediante Decreto $\mathrm{N}^{\circ}$ 0272 del 29 de octubre de 1957). Esta empresa pública fue creada sólo para un periodo de diez años. Luego, en 1968, mediante decreto extraordinario (Decreto N²700), el gobierno creó la Corporación Nacional de Turismo de Colombia, la cual gestionaba con autoridad total los destinos del Turismo y la Hotelería, siendo un organismo descentralizado, dependiente del Ministerio de Desarrollo Económico, y cuyas funciones radican en los siguientes parámetros: a) aprobar planos arquitectónicos hoteleros; b) expedir licencias de funcionamiento; c) clasificar los establecimientos; d) asignar categorías a los hoteles; e) autorizar tarifas; f) reglamentar su operación; g) vigilar su funcionamiento y h) aplicar sanciones. En 1996 se crea el Fondo de Promoción Turística, cuyo objetivo primario es administrar los recursos provenientes de la contribución fiscal, los cuales se destinan exclusivamente a los planes de promoción y marketing turístico y a fortalecer y mejorar la competitividad del sector turístico para incrementar el turismo nacional e internacional, recursos que se someten a los lineamientos de la política turística definidos por el Ministerio de Comercio, Industria y Turismo. También en 1996, y bajo la misma ley (Ley 300 de 1996), se crea el Registro Nacional de Turismo, institución que registra, obligatoriamente, a todas las empresas prestadoras de servicios turísticos en Colombia. Luego, en el año 2003, se crea la Dirección de Turismo (DITUR) (Decreto 210 de 2003), cuyo objetivo primario es la identificación, diseño, formulación y desarrollo de los mecanismos, programas o instrumentos necesarios para la adecuada ejecución de las políticas públicas del sector turístico y su control. DITUR tiene como una de sus tareas relevante la formulación de los planes sectoriales de turismo. Finalmente, y derivado de la última ley del turismo (Ley 1558 de 2012), el Fondo de Promoción Turística se reformula e incluso cambia de nombre, a Fondo Nacional de Turismo, pero mantiene sus objetivos y funciones originales.

Paralelamente a la creación de instituciones públicas nacionales con competencias para gestionar el desarrollo y la reglamentación del turismo, cuya importancia comienza a ser relevante, el gobierno nacional decide también desarrollar leyes que regulen esta incipiente industria. Es entonces cuando en 1968 el gobierno promulga una ley por la cual se establecen estímulos a la industria del turismo (Ley 60 de 26 de diciembre de 1968). Mediante sus primeros dos artículos esta ley presenta el objetivo de fomentar y proteger el turismo que, como fuente generadora de divisas y como actividad que origina trabajo nacional, es industria fundamental para el desarrollo económico del país y será especialmente protegida por el Estado. Y por otro lado, promueve la creación, restauración, conservación, protección, mejoramiento y aprovechamiento de los recursos turísticos nacionales y el fomento del turismo son de utilidad pública e interés social. 
Casi tres décadas después, en 1996, el Gobierno genera la primera Ley General de Turismo (Ley 300 de 1996). Con esta ley el Gobierno da un salto sustancial no sólo en la regulación sino también en el fomento, planificación, desarrollo e impulso de la industria del turismo en Colombia, e incluye regulación sobre protección al consumidor y al medio ambiente. Luego, en el año 2006, esta ley se modifica (mediante la Ley 1101 del 22 de noviembre 2006), pero realiza modificaciones más de forma que de fondo, y sobre todo incluye varios artículos regulatorios en temas fiscales e impositivos. En el año 2006 el gobierno nacional, a través del Plan Nacional de Desarrollo 20062010 (DNP, 2006), establece que las políticas dirigidas al turismo estarán centradas en los lineamientos de los planes sectoriales, y tendrán como objetivo consolidar una oferta competitiva de la industria turística y convertirla en motor de desarrollo regional. Y fija un conjunto de estrategias que deberán desarrollarse en el próximo plan sectorial (2008-2010). En el año 2010, el Plan Nacional de Desarrollo 2010-2014 (DNP, 2010) vuelve a destacar la importancia de la industria turística y desarrolla un conjunto de lineamientos estratégicos en la misma línea del plan anterior pero poniendo gran énfasis en los conceptos de: calidad, institucionalidad y gestión pública, formación y capacitación, e inversión. Finalmente, en 2012 el gobierno promulga una nueva ley, y que está vigente al presente, la Ley 1558 de 10 de Julio de 2012, que modifica las leyes anteriores (Ley 300 de 1996 y Ley 1101 de 2006). Esta última ley del turismo conserva los mismos objetivos y filosofía de las anteriores leyes, de la cual es origen, es decir, el fomento, el desarrollo, la promoción, la competitividad del sector y la regulación de la actividad turística, pero agrega, por primera vez y como novedad, regulación sobre optimización de la calidad turística.

\section{APLICACIÓN DE LAS POLÍTICAS PÚBLICAS Y SUS EFECTOS}

La Dirección de Turismo (del Ministerio de Industria, Comercio y Turismo), desarrolla en el año 2003 el primer plan sectorial de turismo para el periodo 2003-2006 (MinCIT, 2003). En este documento se presenta el estado del arte de la industria, los indicadores turísticos más importantes, la situación de la competitividad, objetivos a cortomedio plazo, ejes temáticos sobre lo que se desarrollará el plan, y finalmente un plan de inversiones. En la Tabla 1 se muestran los principales indicadores turísticos de la época que reflejan el estado del sector por entonces.

TABLA 1. INDICADORES TURÍSTICOS EN COLOMBIA. FUENTE: MINCIT (2003)

\begin{tabular}{|l|c|c|c|c|c|c|}
\hline Indicador & 1996 & 1997 & $\mathbf{1 9 9 8}$ & $\mathbf{1 9 9 9}$ & $\mathbf{2 0 0 0}$ & $\mathbf{2 0 0 1}$ \\
\hline Entrada turistas extranjeros & 630.510 & 639.250 & 674.425 & 546.035 & 557.281 & 664.945 \\
\hline Ocupación hotelera & N.A. & N.A. & $40 \%$ & $39.1 \%$ & $40.8 \%$ & $43.2 \%$ \\
\hline $\begin{array}{l}\text { \% participación del turismo } \\
\text { en el PIB nacional (precios } \\
\text { constantes de 1994) }\end{array}$ & $2,3 \%$ & $2,1 \%$ & $2,09 \%$ & $2,07 \%$ & $2,07 \%$ & $2,08 \%$ \\
\hline $\begin{array}{l}\text { Tasa de crecimiento del } \\
\text { PIB turismo }\end{array}$ & N.A. & $-8,7 \%$ & $-0,48 \%$ & $-0,96 \%$ & $0,0 \%$ & $0,48 \%$ \\
\hline $\begin{array}{l}\text { Divisas generadas por } \\
\text { Turismo (millones USD) }\end{array}$ & 1.120 & 1.043 & 927 & 927 & 1.026 & 1.209 \\
\hline $\begin{array}{l}\text { Tasa de crecimiento de divisas } \\
\text { generadas por turismo }\end{array}$ & N.A. & $-6,88 \%$ & $-11,12 \%$ & $0,0 \%$ & $10,68 \%$ & $17,84 \%$ \\
\hline $\begin{array}{l}\text { Participación del turismo en las } \\
\text { exportaciones de servicios }\end{array}$ & $51,10 \%$ & $48,50 \%$ & $47,50 \%$ & $49,30 \%$ & $51,20 \%$ & $56,10 \%$ \\
\hline $\begin{array}{l}\text { Crecimiento de la participación del } \\
\text { Turismo en las exportaciones }\end{array}$ & N.A. & $-5,09 \%$ & $-2,06 \%$ & $3,79 \%$ & $3,85 \%$ & $9,57 \%$ \\
\hline $\begin{array}{l}\text { Número de empleos directos } \\
\text { generados por el turismo }\end{array}$ & 61.192 & 57.652 & 78.114 & 74.520 & 78.556 & 41.371 \\
\hline $\begin{array}{l}\text { Tasa de crecimiento de generación de } \\
\text { empleo en el sector turismo }\end{array}$ & N.A. & $-5,78 \%$ & $35,5 \%$ & $-4,60 \%$ & $5,42 \%$ & $-47,34 \%$ \\
\hline
\end{tabular}

En lo que se refiere a la oferta turística el informe muestra una importante diversificación de la misma por parte de la industria, donde los principales clusters del sector ya ofrecían al mercado: turismo ecológico, turismo cultural, turismo étnico, turismo rural y turismo recreativo, en ese orden de importancia. En un aspecto positivo, el plan concluye que con respecto a los 7-8 años previos a la formulación del primer plan sectorial, el turismo en Colombia presenta una reactivación y mejoramiento de condiciones competitivas de los principales destinos, una creciente demanda interna, un crecimiento de la ocupación de hoteles en las regiones turísticas, un crecimiento de los vuelos internacionales, un mayor número de movimiento de vehículos por las carreteras, crecimiento de las 
empresas turísticas y del empleo en la industria, y una cada vez mayor inversión extranjera directa en el sector. Pero por el contrario, en cuanto a la competitividad de la industria, el plan sectorial detecta graves problemas y deficiencias en los siguientes aspectos: infraestructuras, capacitación, y planificación, en un primer nivel de importancia, y luego debilidad gremial, promoción, orden público, política turística, gestión pública, calidad y financiación en un segundo nivel de relevancia. Esta situación condujo a la definición de un objetivo claro para el futuro: mejorar la competitividad de los destinos y de los productos turísticos. Y para conseguir este objetivo el plan establece el desarrollo de ciertos 'ejes temáticos': destinos turísticos competitivos, infraestructuras de calidad, seguridad para los viajeros, empresas eficientes, promoción y marketing, formación y capacitación, cooperación internacional, y financiación e incentivos para la inversión.

\subsection{Primer política sectorial de turismo}

En el año 2005 el Consejo Nacional de Política Económica y Social, del Departamento Nacional de Planeación, desarrolla, presenta y aprueba la primer Política Sectorial de Turismo (DNP, 2005). Dicho documento propone los lineamientos para el desarrollo de una política integral del sector turístico y las estrategias a implementar para conseguir tal fin.

Indicadores turísticos. En primer lugar el informe recoge datos relevantes del desarrollo de la industria, considerando que está en pleno desarrollo el plan sectorial de turismo 2003-2006 (analizado en el punto anterior). Todos los grandes indicadores son positivos, ver Tabla 2.

TABLA 2. INDICADORES TURÍSTICOS. FUENTE: DNP (2005)

\begin{tabular}{|l|c|}
\hline Indicador & Dato \\
\hline Crecimiento de la participación del PIB turismo en el PIB nacional (2003-2004) & $105 \%$ \\
\hline Crecimiento de pasajeros vuelos nacionales e internacionales (2003-2004) & $3,4 \% / 13,06 \%$ \\
\hline Crecimiento de desplazamientos vehículos por carretera (2003-2004) & $269 \%$ \\
\hline Crecimiento de pasajeros de terminales terrestres (Bus) (2003-2004) & $127 \%$ \\
\hline Crecimiento (medio anual) de entrada de turistas extranjeros (1999-2004) & $7,7 \%$ \\
\hline Crecimiento (medio anual) de exportaciones turísticas (turismo receptivo) (1999-2004) & $1,17 \%$ \\
\hline
\end{tabular}

Limitaciones competitivas del sector. El documento afirma que a pesar de que Colombia dispone de magníficos escenarios naturales y de variados recursos culturales para el desarrollo turístico, ello no representa grandes ventajas en el momento de conformar los productos turísticos. En otras palabras, el producto turístico nacional no era competitivo en los mercados internacionales debido, principalmente, a la percepción de inseguridad, predominante en los turistas internacionales sobre Colombia en esa época. A nivel interior, el informe detecta los mismos problemas que el plan sectorial de turismo desarrollado en 2003, entre los más importantes: deficiencias en infraestructuras y transportes, difícil acceso a financiación e incentivos por parte de las empresas turísticas, inseguridad, deficiencias en la promoción y comercialización de los productos turísticos, falta de capacitación de personal turístico, carencia de información (estadísticas unificadas del sector), y bajo nivel de utilización de las nuevas tecnologías de la información y comunicación (TIC's).

Estrategias competitivas. Identificadas estas deficiencias en la industria turística, y entendida su importancia e impacto negativo en el desarrollo del turismo, la política sectorial de turismo decidió definir un conjunto de estrategias que intentarían solucionar los problemas detectados a la vez que proyectaría el sector turismo para ubicarlo como uno de los más importantes dentro de la economía nacional. Entonces, con el objetivo de consolidar un ‘nuevo modelo de desarrollo turístico' los esfuerzos de los diferentes agentes económicos se centrarían en seis grandes estrategias: 1) Fortalecimiento institucional necesario para el desarrollo de la actividad. 2) Mejoramiento de las condiciones de seguridad para los viajeros. 3) Establecimiento de una campaña agresiva y sostenida de promoción y marketing (nacional e internacional). 4) Preparación de la oferta turística. 5) Formación del recurso humano y sensibilización turística. 6) Consolidación de un sistema de información turística que permita formular políticas de gobierno y el uso de tecnologías de la información.

Recomendaciones. Finalmente el documento de política sectorial de turismo propone un conjunto de recomendaciones. De entre todas ellas las más relevantes a destacar, incluso como novedad con respecto a informes y planes anteriores, son la solicitud a otras instituciones públicas del gobierno central (ministerios y otras grandes agencias), con impacto directo e indirecto en la industria del turismo, a realizar planes, programas y un conjunto de actividades dirigidas a dar soporte al desarrollo y ejecución de las estrategias propuestas por la política sectorial del turismo. 


\subsection{Segundo plan sectorial de turismo}

El segundo plan sectorial de turismo, 2008-2010 (MinCIT, 2008), se desarrolló y presentó a mediados de 2008 cuando, a nivel de políticas públicas del sector, se tenía ya terminado y cerrado el plan sectorial de turismo $2003-$ 2006, ya se había formulado la primer política sectorial de turismo (2005), y estaba en vigor un nueva ley de turismo aprobada en 2006.

Situación. Este nuevo plan sectorial reconoce que el desarrollo de políticas turísticas, iniciadas apenas cinco años atrás, ha sido factor fundamental para mantener un esfuerzo sostenido hacia la consolidación de Colombia como un país con un destino turístico bien estructurado y con aceptables niveles de competitividad. El plan ya empieza a identificar importantes avances relativos si se compara con otros países de la región. Las variables macro - inseguridad, imagen negativa del país, economía con niveles modestos de crecimiento llevaron a centrar los esfuerzos en la recuperación de un turismo interno antes que pensar en conquistar turismo receptivo. Diversificación de producto, irrupción de nuevos destinos y aplicación de estrategias de desarrollo regional ordenado, marcaron esta etapa. Hasta la fecha la estrategia macro ha estado centrada en mejorar la competitividad de los productos y de los destinos turísticos. Los resultados de este proceso de consolidación del turismo interno y el inicio de la recuperación del turismo receptivo permiten constatar que el sector empieza a ganar un espacio significativo en las expectativas del país y en la economía colombiana. Los indicadores más importantes (entradas de viajeros extranjeros, empleo generado, aporte al PIB y ocupación hotelera) muestran que el sector está atravesando por un buen momento. En definitiva, el plan sectorial 2008-2010 concluye que a pesar de los buenos resultados registrados en esa época, consecuencia directa de las políticas públicas turísticas, aun queda mucho por mejorar, sobre todo en los estándares de competitividad.

Indicadores. Todos los indicadores turísticos típicos muestran un crecimiento significativo al compararlos a periodos próximos anteriores, cuando aun no estaban en plena ejecución las políticas turísticas o recién empezaban a implementarse. Entrada de turistas extranjeros no-residentes, empleo directo generado en la industria, crecimiento del PIB turismo y ocupación hotelera, todos ellos presentan una evolución positiva en el periodo analizado (ver Tabla 3).

TABLA 3. INDICADORES TURÍSTICOS. FUENTE: MINCIT (2008)

\begin{tabular}{|l|c|}
\hline Indicador & Dato \\
\hline Crecimiento de entrada de turistas extranjeros (2000-2006) & $89 \%$ \\
\hline $\begin{array}{l}\text { Crecimiento empleos directos en turismo (comparativa de medias entre } \\
\text { los periodos 1995-2000 y 2000-2005) }\end{array}$ & $-16.8 \% / 31.6 \%$ \\
\hline $\begin{array}{l}\text { Crecimiento del PIB turismo (comparativa de media anual entre los } \\
\text { periodos 1998-2002 y 2003-2006) }\end{array}$ & $0.58 \% / 6.35 \%$ \\
\hline $\begin{array}{l}\text { Crecimiento ocupación hotelera (comparativa de media anual entre los } \\
\text { periodos 2000-2002 y 2003-2006) }\end{array}$ & $42.5 \% / 52.7 \%$ \\
\hline
\end{tabular}

Competitividad. A la fecha, 2008, la baja competitividad de la industria sigue siendo un problema y a la vez un gran desafío por alcanzar por parte de las autoridades turísticas colombianas. Esta baja competitividad del turismo es coherente con la baja competitividad general del país, donde en 2006 Colombia ocupaba el puesto 65 de 125 evaluados en el informe de competitividad global del Foro Económico Mundial (WEF, 2006). En el año 2007 el Foro Económico Mundial realiza el primer informe de medición de la competitividad para el sector viajes y turismo (WEF, 2007). De una muestra de 124 países Colombia ocupa el puesto 72. La evaluación toma como referencia las principales variables que inciden para que un país establezca o no condiciones favorables para el desarrollo del sector. El informe revela fortalezas de Colombia con respecto a la no exigencia de visas, la buena capacitación de las personas vinculadas al sector, el precio del combustible de aviación y en la cantidad de aeropuertos. Entre las mayores deficiencias están las derivadas de la seguridad, impuestos y tarifas aeroportuarias e impacto de la tributación en la industria turística.

Objetivo y estrategias. El objetivo principal de este plan sectorial fue consolidar los procesos regionales de turismo de tal manera que se dispusiera de una oferta de productos y destinos altamente competitivos para los mercados nacional e internacional. Para el logro de los objetivos y metas propuestos, el plan fijó la aplicación de siete estrategias centrales: 1) mejoramiento y consolidación de la competitividad, 2) mejoramiento de la infraestructura turística, incluyendo una mejor y mayor conexión de los destinos turísticos, 3) fortalecimiento del marketing, la promoción y la comercialización, 4) creación de un sistema de información turística, 5) implementación de estímulos a la demanda y a la oferta, 6) disponer recursos para apoyar iniciativas productivas turísticas de las 
comunidades, 7) educación para fortalecer la cultura turística. Cada una de las estrategias se desplegaría través de programas y acciones.

\subsection{Tercer plan sectorial de turismo}

El plan sectorial de turismo 2011-2014, tercero de su tipo, se presenta a mediados de 2011 (MinCIT, 2011) para dar continuidad al desarrollo de las políticas públicas del turismo. Este plan se concentra en reforzar algunos elementos ligados al último plan sectorial de turismo (2008-2010) y a consolidar los principales lineamientos del sector que están registrados en el Plan Nacional de Desarrollo 2010-2014 (DNP, 2010).

Estado de la industria. Para la fecha, 2011, el sector turístico en Colombia pasa por un buen momento y está cada vez más fortalecido. Se sigue de forma rigurosa las políticas públicas, se han canalizado esfuerzos públicos y privados para generar espacios de desarrollo para las iniciativas turísticas, los lineamientos de las políticas sectoriales de turismo han contribuido progresivamente a afianzar el compromiso de los diversos entes del Estado para apoyar al sector; la reforma de la Ley 300 de 1996 a través de la Ley 1101 de 2006 generó mayores herramientas y recursos para la promoción del sector, y finalmente el Ministerio de Comercio, Industria y Turismo se enfocó en el desarrollo de iniciativas para fortalecer la competitividad y sostenibilidad del sector desde cuatro ámbitos fundamentales: a) el desarrollo de oferta turística más competitiva; b) mejorar el acceso y la conectividad con los mercados turísticos; c) fortalecimiento de la promoción turística internacional y nacional y d) la coordinación público-privada y nacional-regional para el desarrollo turístico.

Comportamiento de los indicadores. Para la época, el turismo colombiano está experimentando un proceso de crecimiento que no se había registrado en toda su historia. El turismo receptivo reaccionó favorablemente a las políticas diseñadas por el gobierno, y empezó a mostrar un importante crecimiento del número de visitantes extranjeros que ingresan a Colombia. Como resultado de los programas y proyectos para el mejoramiento de la oferta turística, la competitividad del sector y la promoción nacional e internacional, en el año 2010 la entrada de turistas extranjeros a Colombia se incrementó en 1,4 millones con respecto al año 2002, crecimiento del $154 \%$ en el periodo. Como consecuencia del incremento de turistas extranjeros, el ingreso de divisas al país pasó de USD 1,2 mil millones en 2002 a USD 2,6 mil millones en 2009, un incremento del 115\%, ubicándose el turismo como el tercer exportador (en generación de divisas) después del petróleo y el carbón. En el contexto mundial se empieza a tener una imagen más positiva del país, y por otro lado las grandes organizaciones internacionales del sector comienzan a reconocer el trabajo de las autoridades turísticas colombianas. Un estudio titulado Colombia, back on the map of world tourism (UNWTO, 2009), publicado en enero de 2009, hace referencia a las diferentes acciones emprendidas por las autoridades colombianas, tanto a nivel nacional como internacional, para dinamizar su industria turística. Y en octubre de 2009 el estudio Handbook on Tourism Destination Branding (UNWTO \& ETC, 2009) resaltó la importancia del cambio positivo de imagen de Colombia, lo cual, por supuesto, ha repercutido favorablemente en la actividad turística.

Objetivos y estrategias. El objetivo primario para el periodo es mejorar la competitividad de los servicios y destinos turísticos de Colombia con el fin de hacer del turismo una estrategia de desarrollo sostenible para el país, que contribuya a la generación de empleo, a la prosperidad de las regiones. Las estrategias a desarrollar son conceptualmente las mismas que en el plan sectorial anterior, pero esta vez están más particularizadas a objetivos específicos concretos, con el énfasis en los siguientes temas: dinamizar la cooperación internacional, más programas para la mejora de la competitividad, calidad turística, seguridad, formación y capacitación, mejora de las infraestructuras de soporte al turismo, adaptabilidad del sistema aeroportuario y del transporte aéreo a las necesidades turísticas, programas de promoción de inversión extranjera directa en turismo, diversificación en la oferta turística con el desarrollo de productos turísticos especializados, y finalmente continuar con la promoción del turismo colombiano a nivel nacional e internacional.

\subsection{Cuarto plan sectorial de turismo}

En agosto de 2014 el gobierno, a través del Ministerio de Comercio, Industria y Turismo y el Departamento Nacional de Planeación, presenta el cuarto plan sectorial de turismo para el periodo 2014-2018 (MinCIT, 2014). Este plan da continuidad a lo exigido por el marco normativo (leyes de turismo) y documentos de política sectorial, como ya se viene haciendo, de forma ininterrumpida, desde principios de la anterior década.

Situación de la industria turística colombiana. El informe destaca que, a la fecha, el turismo en Colombia es una industria de honda repercusión social, cultural y económica. Y por otro lado, ya se puede afirmar que Colombia está inserta en la dinámica mundial del turismo. Por ello las cifras actuales son reveladoras. La producción del sector de hoteles y restaurantes, las llegadas de turistas extranjeros los ingresos de la cuenta de viajes de la balanza de pagos, la inversión extranjera directa en la industria turística, y el dinamismo que registra la construcción de 
hoteles en los últimos años viene presentando importantes crecimientos. Como resultado, el PIB turismo fue uno de los de mayor crecimiento en el último cuatrienio (incluso por encima del PIB nacional), después de minas, construcción, servicios financieros y transporte.

Indicadores. El PIB del turismo después de minas, construcción, servicios financieros y transporte fue uno de los sectores de mayor crecimiento en el periodo 2010-2013. En 2013 la participación del PIB turismo en el PIB nacional fue del 12\%; el crecimiento del PIB turismo en el 2013, con respecto al 2012, fue del 4,6\% (MinCIT, 2015). La balanza de pagos 'cuenta de viajes y transporte de pasajeros', concepto que equivale a turismo receptivo en Colombia, es decir 'exportaciones de servicios turísticos' o en otros términos 'ingreso de divisas por turismo', presenta un acelerado y dinámico crecimiento en la última década, alcanzando en el año 2013 la cifra de USD 4.758 millones, aproximadamente un $400 \%$ más que en el año 2003 cuando se inician las políticas sectoriales de turismo. En otro orden, hoy en día la industria del turismo en Colombia es el primer generador de empleo, ya que absorbe algo más de la cuarta parte del total de empleos generados en Colombia (27,45\%) (DANE, 2014b). Más específicamente, el número de empleados en el sector alcanzó la cifra de seis millones en el año 2013, casi dos millones más que en el año 2002. La generación de empleo el sector del turismo ha experimentado un comportamiento similar (e incluso superior) al de generación de empleo a nivel nacional, y con crecimientos siempre positivos desde el 2008 (DANE, 2014). Un indicador que registró gran dinamismo en los últimos cuatro años fue la construcción de hoteles, cuando ingresaron al mercado 726.088 metros cuadrados de áreas culminadas con destino a hoteles y se encontraban $8.116 .548 \mathrm{~m} 2$ más en proceso de construcción, siendo 2010 el año de mayor dinamismo con un crecimiento de 78,1\% (MinCIT, 2014). Por otro lado, la tendencia de crecimiento de la llegada de turistas extranjeros a Colombia ha sido positiva y constante con un promedio anual de incremento del 13,8\% en el período $2004-2013$ y un promedio anual de llegadas de 1.451 .360 turistas. Esto demuestra que los acontecimientos mundiales como la última crisis económica internacional de 2008-2009, no han afectado significativamente la llegada de turistas al país. En 2013 Colombia registró un incremento en los flujos de llegadas de extranjeros no residentes superior al promedio mundial. Mientras que de 2012 a 2013 el promedio de crecimiento en las llegadas en el mundo fue de $4,5 \%$, en Colombia fue de $8,5 \%$ es decir 4 puntos porcentuales superior al crecimiento de las economías avanzadas y emergentes (MinCIT, 2014; UNWTO, 2014). La ocupación hotelera tuvo un crecimiento moderado, pero positivo, en el periodo 2010-2013, con una media de 0,9\% para todo el periodo. Y finalmente, en cuanto al movimiento de pasajeros por los tres modos, aéreo, marítimo y terrestre, los indicadores muestran un importante crecimiento en el periodo 2002-2013 (ver Tabla 4).

TABLA 4. MOVIMIENTO DE PASAJEROS. FUENTE: MINISTERIO DE TRANSPORTE (2014)

\begin{tabular}{|l|c|}
\hline Indicador & Dato \\
\hline Crecimiento de pasajeros terrestres domésticos (bus, auto) (periodo 2002-2013) & $80 \%$ \\
\hline Crecimiento de pasajeros aéreos nacionales (periodo 2002-2013) & $137 \%$ \\
\hline Crecimiento de pasajeros aéreos internacionales (periodo 2002-2013) & $210 \%$ \\
\hline Crecimiento de pasajeros intl. marítimos (vía cruceros) (periodo 2002-2013) & $251 \%$ \\
\hline
\end{tabular}

Competitividad. La competitividad sigue siendo el gran problema a solucionar por las autoridades turísticas colombianas. Al margen de los excelentes indicadores económicos que presenta la industria, las políticas públicas sectoriales desarrollas a lo largo de una década, no han sido capaces de generar avances relevantes en temas de competitividad. El análisis en este tema del presente plan sectorial se remite por completo al informe de competitividad del Foro Económico Mundial (WEF, 2013). Según este informe Colombia, en el 2012, obtuvo una calificación de 3,9 lo que represento 0,04 puntos por debajo de la obtenida en $2011(3,94)$ y un retroceso de siete puestos en el ranking mundial con relación al mismo año. A nivel de continente americano, en 2012 Colombia se encontraba en el puesto quince entre veinticuatro países, y en Suramérica entre diez países Colombia ocupó séptimo puesto.

Objetivos estratégicos. El presente Plan Sectorial de Turismo 2014-2018 propone cuatro objetivos estratégicos (DNP, 2014):

1. Impulsar el desarrollo y consolidación de destinos y productos turísticos competitivos y sostenibles a nivel regional y territorial, a partir del aprovechamiento responsable de la diversidad natural, étnica y cultural. Acciones claves: a) desarrollo de productos turísticos, b) generar infraestructuras para el turismo, c) mejorar y promover la calidad y productividad empresarial, d) promover el turismo social y responsable.

2. Impulsar la conectividad para el turismo del país a nivel regional, nacional e internacional. Acciones claves: a) fortalecer las gestiones de mejoramiento de las condiciones de acceso a los atractivos turísticos, b) 
apoyar las gestiones para la facilitación de viajes que favorezcan la competitividad de los destinos, c) realizar estudios de conectividad aérea, terrestre fluvial y marítima, que identifique las necesidades reales en competitividad de los destinos, d) promover el mejoramiento de los servicios en terminales aéreos, terrestres, marítimos y fluviales, e) gestionar medios de acceso a los destinos turísticos en concordancia con sus mercados emisores.

3. Promocionar turísticamente a Colombia a nivel regional, nacional e internacional con estrategias efectivas e innovadoras. Acciones claves: a) promoción del turismo doméstico, b) promoción y marketing internacional.

4. Mejorar la articulación institucional nación-región, con la participación del sector privado, público y comunitario, y desarrollar mecanismos que promuevan la gestión eficiente del turismo. Acciones claves: a) fortalecer el sistema nacional de turismo, b) fortalecer la cooperación internacional para ofertar fortalezas y recibir transferencia de conocimiento y recursos, para el desarrollo turístico, c) participar activamente en los organismos internacionales de turismo y redes de conocimiento, d) difundir el marco normativo del turismo, e) fortalecer los canales de comunicación entre el Ministerio de Comercio, Industria y Turismo y las entidades de gestión del turismo a nivel territorial, f) establecer mecanismos de seguimiento al uso adecuado y sostenible de las inversiones en infraestructura, g) promover altos niveles de formación para los responsables tanto de las acciones de gestión como de la administración y manejo de las infraestructuras turísticas, h) fortalecer los sistemas de información turística nacional y regional, así como el plan estadístico sectorial de turismo.

Finalizando, el plan sectorial 2014-2018 reconoce (aunque escapa al alcance del desarrollo de las estrategias del presente plan) que la consecución de la paz juega un papel fundamental en el despegue definitivo del turismo, como así también de todas las industrias del país y en definitiva de Colombia. Al tiempo de edición de este trabajo el gobierno colombiano está conduciendo el proceso de negociación de la paz en La Habana, Cuba (Presidencia de la República, 2015).

\section{COMPETITIVIDAD EN EL TURISMO COLOMBIANO}

\subsection{El concepto de competitividad en el turismo}

En el campo de la investigación del turismo la competitividad de los destinos puede ser definida como la capacidad de un país para crear valor añadido y, por tanto, mejorar el bienestar nacional mediante la gestión de las prestaciones y los procesos y la integración de las relaciones entre ellos en un modelo económico y social (Crouch y Ritchie, 2001). Hassan (2000) define la competitividad del turismo como la capacidad de un destino para crear e integrar al valor agregado bienes que sostendrían los recursos locales y preservar su posición en el mercado frente a sus competidores. Para analizar la competitividad de los destinos turísticos, Crouch y Ritchie (1999) desarrollaron el modelo (de competitividad) de Calgary. Para dicho modelo la ventaja competitiva (concepto basado en el modelo de Porter (1990)) se refiere a la capacidad de un destino turístico de utilizar sus recursos de manera eficiente a medio y largo plazo. Por lo tanto, un destino turístico cuyos habitantes están convencidos de la posibilidad del crecimiento económico a través de la explotación de sus recursos, que conocen tanto sus fortalezas como sus debilidades y que desarrollan una adecuada política de marketing, sin duda transformarán dicho destino turístico en uno más competitivo. Por otro lado, Dwyer y Kim (2003a, 2003b) proponen un modelo de competitividad de carácter integrado, que considera que los recursos heredados, los recursos creados y los recursos de apoyo son los principales componentes en el éxito de un destino turístico y es la base de la competitividad turística. Este modelo se complementa con las condiciones de situación, así como del destino y de la gestión de la demanda. Todos estos factores se aúnan para lograr la competitividad en los destinos turísticos, cuyo objetivo final es mejorar la calidad de vida y bienestar de sus habitantes. En definitiva, los estudios teóricos sobre la competitividad del turismo destacan los siguientes factores: las políticas públicas, la inversión extranjera directa, la promoción del turismo, los recursos culturales, los recursos humanos, los recursos naturales, infraestructura, calidad y precio (Molina et al., 2014).

\subsection{Políticas públicas dirigidas a la competitividad turística en Colombia}

Se ha mencionado en los análisis previos que la competitividad es uno de los aspectos más relevantes a mejorar en el sector/industria del turismo colombiano. Ahora bien, el gobierno colombiano, consciente de esta problemática, viene trabajando desde hace años en mejorar los estándares de competitividad, y en todos los sectores productivos. Por ello, se cree necesario hacer un rápido análisis de las políticas públicas en materia de competitividad, muchas de las cuales afectan de forma directa e indirecta al sector turismo. 
Entonces, desde la apertura comercial de Colombia, iniciada en 1991, hasta hoy se han implementado diferentes políticas, programas y leyes para aumentar la productividad y la competitividad de los diferentes sectores productivos, tanto en las regiones como a nivel nacional. El enfoque, la estructura, la directiva y el nombre de cada iniciativa han cambiado de acuerdo con cada gobierno. Sin embargo, en la práctica los objetivos han sido similares: aumentar la competitividad del aparato productivo del país. A continuación se hace un resumido análisis de las principales iniciativas implementadas en las últimas dos décadas para mejorar la competitividad en el país. Las políticas nacionales de competitividad emanan de los diferentes planes nacionales de desarrollo formulados cada cuatro años.

En el periodo 1990-1994, el plan nacional de desarrollo 1990-1994 (DNP, 1990) define ciertas políticas en materia de competitividad, a saber:

- se eliminaron o redujeron drásticamente muchos de los instrumentos e instituciones dedicadas al proteccionismo;

- se redujeron los subsidios;

- se ordenó el desarrollo de estudios sobre la competitividad sectorial en diferentes sectores productivos;

- en 1990 se promulga la Ley de Ciencia y Tecnología para la promoción de la labor investigativa;

- las Zonas Francas fueron promovidas como elemento para la atracción de inversión y dinamismo productivo;

En el periodo 1994-1998, a través del plan nacional de desarrollo 1994-1998 (DNP, 1994), se realizaron importantes avances en cuanto a la formulación de una estrategia integral de competitividad, bajo la cual se creó un nuevo marco institucional con el propósito de crear una cultura exportadora basada en la productividad. Las principales políticas en este periodo fueron:

- se formula la Primera Estrategia Nacional de Competitividad;

- se creó el Consejo Nacional de Competitividad;

- se crearon los Acuerdos Sectoriales de Competitividad;

- comienza a operar la Política Nacional de Ciencia y Tecnología:

- se formuló un estudio llamado 'Consejo de Sabios', el cual concluyó que el énfasis y primer obstáculo a superar en materia de competitividad era el bajo nivel de la educación superior y el bajo presupuesto para la investigación y el desarrollo.

En el periodo 1998-2002, a través del plan nacional de desarrollo 1998-2002 (DNP, 1998), se plantearon dos estrategias complementarias que tomaban elementos de la estructura institucional propuesta en el plan anterior y formulaba un enfoque más explícito basado en políticas de asociatividad. Las políticas más relevantes del plan fueron:

- se diseña y formula el (primer) Plan Estratégico Exportador 1999-2009, desde el cual se plantearían las bases de la estrategia para el desarrollo del sector productivo;

- se formula la Política Nacional para la Productividad y la Competitividad;

- se crearon 32 Comités Público Privados Regionales y 34 Convenios de Competitividad Exportadora de Cadenas Productivas.

En el periodo 2002-2006, a través del plan nacional de desarrollo 2002-2006 (DNP, 2002), las políticas sobre competitividad vienen enmarcadas (y guiadas) con el inicio de las negociaciones del TLC con Estados Unidos, las cuales dieron origen al tratado que se implementó una década después. Las políticas se formulan con miras a preparar el aparato productivo nacional a las condiciones y exigencias del mercado estadounidense. Las políticas más relevantes en materia de competitividad fueron:

- se rediseña tanto el Sistema como la Comisión Nacional de Competitividad;

- se crea el Consejo Privado de Competitividad;

- se realiza una optimización de los instrumentos de desarrollo empresarial;

- se crea una Alta Consejería para la Competitividad.

En el periodo 2006-2010, a través del plan nacional de desarrollo 2006-2010 (DNP, 2006), el gran hito de este periodo fue la formulación del Plan Nacional de Competitividad y sus cinco pilares: desarrollo de clúster 
de clase mundial, salto en productividad, formalización empresarial, fomento e innovación, y estrategias transversales de promoción de competencia en inversión. En este periodo de crea el Consejo Privado de Competitividad, y se impulsan los incentivos sectoriales, los apoyos a clusters, y los planes regionales de competitividad.

En el periodo 2010-2014, a través del plan nacional de desarrollo 2010-2014 (DNP, 2010), las principales políticas en competitividad fueron:

- se definen nuevos objetivos para el Sistema Nacional de Competitividad;

- se ampliaron los sectores cobijados por el Programa de Transformación Productiva;

- se destinaron recursos para la investigación y el desarrollo, los proyectos de infraestructura regionales y la productividad industrial respectivamente;

- se lanzó el Plan de Impulso a la Prosperidad y al Empleo (PIPE); este paquete de programas incluye herramientas para estimular la competitividad de la industria a nivel de las firmas, para lo cual se disminuyó el costo del capital, se plantearon mejores condiciones tributarias y se invirtió en infraestructura y estabilidad para el sector agropecuario.

Para el periodo 2014-2018, según el plan nacional de desarrollo 2014-2018 (DNP, 2014), las estrategias en materia de competitividad se encuentran contempladas en la Agenda Nacional de Competitividad 2014-2018, la cual está orientada a generar un mayor valor económico y social, y a mejorar las condiciones para el desarrollo de actividades empresariales por parte de los más diversos agentes (empresas privadas, organizaciones públicas, instituciones y organizaciones sociales, etc.). Esta Agenda se enfoca en once ejes prioritarios alrededor de los cuales se definirán proyectos cuyos avances requerirán de un importante esfuerzo de articulación al interior del gobierno, así como de las esferas pública y privada. Estos ejes prioritarios son: i) Ciencia, tecnología e innovación; ii) Desarrollo regional; iii) Infraestructura; iv) Transformación agropecuaria; v) Transformación industrial; vi) Educación, empleo y salud; vii) Institucionalidad; viii) Estabilidad macroeconómica; ix) Justicia; $x$ ) Lucha contra la ilegalidad y la corrupción; y, xi) Prácticas leales de competencia.

\section{RESULTADOS EN EVOLUCIÓN DEL CUARTO PLAN SECTORIAL DE TURISMO}

A la fecha de edición del presente artículo, segundo trimestre de 2015, se está recién en el primer año de ejecución del actual plan sectorial de turismo 2014-2018, sin embargo ya se tienen los datos consolidados (de los años 2013 y 2014) de los principales indicadores del sector que se detallan en la Tabla 5 (MinCIT, 2015).

TABLA 5. EVOLUCIÓN RECIENTE DE LOS PRINCIPALES INDICADORES TURÍSTICOS. FUENTE: MINCIT (2015)

\begin{tabular}{|l|c|}
\hline Indicador & Dato \\
\hline Turismo receptivo, crecimiento 2014-2013 & $11,9 \%$ \\
\hline Entrada de turistas intl., crecimiento 2014-2013: vía aérea / vía cruceros & $12,1 \% / 2,4 \%$ \\
\hline Turismo emisor, crecimiento 2014-2013 & $7,5 \%$ \\
\hline Turismo interior: pasajeros aéreos domésticos, crecimiento 2014-2013 & $6,2 \%$ \\
\hline Ocupación hotelera, años 2014 / 2013 & $53 \% / 52,7 \%$ \\
\hline Personal ocupado en hoteles, crecimiento 2014-2013 & $4,4 \%$ \\
\hline Agencias de viajes: ingresos nominales, crecimiento 2014-2013 & $7,6 \%$ \\
\hline Agencias de viajes: personal ocupado, crecimiento 2014-2013 & $7,3 \%$ \\
\hline Participación del PIB turismo en el PIB nacional, año 2014 / 2013 & $12 \% / 12 \%$ \\
\hline IED, $\%$ de participación del turismo en el total nacional, 2014 / 2013 & $5,2 \% / 7 \%$ \\
\hline $\begin{array}{l}\text { Balanza de pagos: viajes y transporte de pasajeros, participación en el } \\
\text { total de exportaciones de servicios, 2014 / 2013 }\end{array}$ & $71,8 \% / 69,3 \%$ \\
\hline $\begin{array}{l}\text { Balanza de pagos: viajes y transporte de pasajeros, crecimiento de las } \\
\text { exportaciones, 2014-2013 }\end{array}$ & $4,7 \%$ \\
\hline $\begin{array}{l}\text { Balanza de pagos: viajes y transporte de pasajeros, crecimiento de las } \\
\text { importaciones, 2014-2013 }\end{array}$ & $15,9 \%$ \\
\hline $\begin{array}{l}\text { Construcción de hoteles: crecimiento de áreas (m2) en proceso / culminadas } \\
\text { periodo 2014-2010 }\end{array}$ & $101 \% / 58 \%$ \\
\hline
\end{tabular}


En otro orden, en cuanto a la evolución de los indicadores de competitividad a nivel internacional, comparando los resultados del último (y actual) informe sobre competitividad turística del Foro Económico Mundial (WEF, 2015) con el anterior informe (WEF, 2013), se tiene para Colombia una evolución relativa positiva (ver Tabla 6). Según los resultados del informe, en Colombia se ha mejorado, ya sea en puntuación y/o en posición en el ranking global, en la mayoría de indicadores. Sin embargo, se verifica un retroceso en los indicadores de: seguridad (éste muy acentuado), mercado laboral, y recursos naturales.

TABLA 6. INDICADORES DE COMPETITIVIDAD TURÍSTICA INTERNACIONAL; PUNTUACIÓN: 1-7, PAÍ́SES EVALUADOS: 140 (2012), 141 (2014). FUENTE: WEF $(2015,2013)$

\begin{tabular}{|l|c|c|}
\hline Indicador & $\begin{array}{c}\mathbf{2 0 1 2} \\
\text { Puntaje / Posición }\end{array}$ & $\begin{array}{c}\mathbf{2 0 1 4} \\
\text { Puntaje / Posición }\end{array}$ \\
\hline Política y regulaciones (apertura para los negocios) & $4,6 / 56$ & $4,3 / 8$ \\
\hline Sostenibilidad medioambiental & $4,3 / 97$ & $3,9 / 87$ \\
\hline Seguridad & $3,8 / 115$ & $5,8 / 140$ \\
\hline Salud e higiene & $3,6 / 100$ & $4,3 / 92$ \\
\hline Priorización de viajes y turismo & $4,2 / 88$ & $2,7 / 70$ \\
\hline Infraestructuras de transporte aéreo & $2,9 / 73$ & $2,5 / 124$ \\
\hline Infraestructuras de transporte terrestre y portuario & $2,6 / 131$ & $3,6 / 90$ \\
\hline Infraestructuras turísticas & $2,8 / 93$ & $4,2 / 67$ \\
\hline Infraestructuras TIC's & $2,9 / 77$ & $4,5 / 86$ \\
\hline Competitividad en precios (industria turística) & $4,2 / 105$ & $4,4 / 79$ \\
\hline Recursos humanos y mercado laboral & $4,9 / 72$ & $4,1 / 24$ \\
\hline Recursos naturales & $5,1 / 16$ & $3,2 / 24$ \\
\hline Recursos culturales & $3,5 / 37$ & \\
\hline
\end{tabular}

\section{CONCLUSIONES}

Hace cinco décadas no existía en Colombia una industria del turismo como tal, en toda su dimensión económica, social e institucional. Es recién en 1968 cuando el gobierno, vía ley, establece los primeros estímulos al turismo y crea, también por decreto ley, la Corporación Nacional de Turismo de Colombia, cuyo objetivo era gestionar las actividades de los hoteles y ciertas actividades en los destinos turísticos. Luego, el sector tendrá que esperar hasta el año 1996 cuando se dicta la primera ley general de turismo. Se puede afirmar que con esta ley el gobierno concede status formal de industria al turismo en Colombia. A partir de esta ley el gobierno puede regular la industria como así también gestionar y controlar su fomento, planificación, desarrollo e impulso. El siguiente hito relevante en la historia del desarrollo de la industria turística es en el año 2003, cuando el gobierno crea la Dirección de Turismo (DITUR), dependiente del Ministerio de Industria, Comercio y Turismo, cuyo objetivo primario fue la identificación, diseño, formulación y desarrollo de los mecanismos, programas o instrumentos necesarios para la adecuada ejecución de las políticas públicas del sector turístico y su control. En otras palabras, esta institución tiene como una de sus tareas relevantes la formulación de los planes sectoriales de turismo. A partir del año 2003, y en colaboración con el Departamento Nacional de Planeación de Colombia, se desarrollan cuatro planes sectoriales de turismo, para los periodos 20032006, 2008-2010, 2011-2014 y 2014-2018. Paralelamente, y considerando la creciente evolución de la industria turística, los últimos Planes Nacionales de Desarrollo, elaborados cada cuatrienio, incluyen al turismo como sector estratégico de la economía colombiana. En esta línea, en el año 2005 el Consejo Nacional de Política Económica y Social, del Departamento Nacional de Planeación, desarrolla, presenta y aprueba la primer Política Sectorial de Turismo. Dicho documento propone los lineamientos para el desarrollo de una política integral del sector turístico y las estrategias a implementar.

Los objetivos y estrategias de los diferentes planes sectoriales turísticos han ido evolucionado y adaptándose en función tanto de los logros conseguidos por las políticas implementadas, como así también de la propia evolución natural de la industria, a nivel doméstico, regional y mundial, que fijaba los nuevos retos y desafíos a alcanzar. En ese sentido la positiva evolución de casi todos los indicadores de la industria en la última década no dejan lugar a dudas sobre el éxito de las políticas públicas turísticas, sobre todo si se tiene en cuenta el corto tiempo (sólo 10 años) en el que la industria del turismo pasa a situarse en una posición relevante en la economía colombiana, hoy en día el primer generador de empleo del país y el tercer exportador en divisas. 
Asimismo, a pesar de las excelentes metas alcanzadas, gracias a los esfuerzos realizados por el gobierno y las instituciones (públicas y privadas), desarrollando y dando obligada continuidad a la aplicación de las políticas públicas, las autoridades turísticas aún tienen pendiente, principalmente, dos grandes desafíos a alcanzar, la competitividad y la seguridad. Empezando por esta última, el gobierno reconoce que la consecución de la paz dará un impulso inigualable al turismo, no sólo a nivel doméstico sino que también situará al turismo colombiano en el escenario regional y mundial del turismo. Por ello el gobierno nacional conduce desde el año 2012 las negociaciones de paz en La Habana, Cuba, para dar una salida negociada al conflicto interno armado con el principal grupo armado terrorista, en lucha desde hace medio siglo, consciente de que la paz mejorará y potenciará la percepción de seguridad de los turistas extranjeros y abrirá definitivamente las puertas al turismo internacional. $Y$ en cuanto a la competitividad los diferentes planes sectoriales han ido mejorando y reforzando en sus planteamientos en este tema, sin aun poder alcanzar estándares competitivos de nivel internacional, tanto a nivel regional como mundial. Pero las autoridades públicas turísticas reconocen que para conseguir altos niveles de competitividad y calidad se debe seguir trabajando en planes de formación, capacitación y entrenamiento, en todos los órdenes y con todos los agentes del turismo, tanto públicos como privados, además de apoyarse en las diferentes políticas públicas en competitividad, en curso desde hace dos décadas.

En definitiva, y concluyendo, si como es de esperar, se sigue dando continuidad a la ejecución de las políticas públicas turísticas iniciadas hace una década, el turismo colombiano está llamado a continuar jugando un importante papel en el desarrollo social y económico de Colombia.

\section{REFERENCIAS}

Aguilar, L.F. (1996). El estudio de las políticas públicas. México D.F.: Ed. M.A.P.

Álvarez Díaz, A.E. (1992). Análisis de políticas públicas. Caracas: CLAD.

Bayón, F. (1999). 50 años del Turismo Español. Un análisis histórico y estructural. Madrid: Centro de Estudios Ramón Areces S.A.

Benavides, G.F. (2015). "Las políticas públicas del turismo receptivo colombiano". Suma de Negocios, 6(13), 66-73. DOI: http://dx.doi.org/10.1016/j.sumneg.2015.08.005

Benavides, G.F. y Venegas, S.P. (2013). "Una aproximación a la competitividad, las tendencias y la política pública en el turismo colombiano". Revista de Análisis Turístico, 16, 1-12.

Berno, T. (1996). "Cross-cultural research methods: Content or context? A Cook Islands example". In R. Butler, \& T. Hinch (Eds.), Tourism and indigenous peoples. London: International Thomson Business Press.

Crouch, G.I. y Ritchie, J.R.B. (1999). "Tourism, competitiveness and societal prosperity". Journal of Business Research, 44(3), 137-152. DOI: 10.1016/S0148-2963(97)00196-3

DANE - Departamento Administrativo Nacional de Estadísticas de Colombia - (2014). Cuentas Nacionales Departamentales de Colombia. En línea: <https://www.dane.gov.co/files/investigaciones/pib/ departamentales/B_2005/Ramas_actividad_2011def_2013pre.xls> (consulta: 8 de junio 2015).

DNP (2014). Plan Nacional de Desarrollo 2014-2018. Bogotá: Departamento Nacional de Planeación.

DNP (2010). Plan Nacional de Desarrollo 2010-2014. Bogotá: Departamento Nacional de Planeación.

DNP (2006). Plan Nacional de Desarrollo 2006-2010. Bogotá: Departamento Nacional de Planeación.

DNP (2005). Política Sectorial de Turismo. Documento CONPES 3397. Bogotá: Departamento Nacional de Planeación.

DNP (2002). Plan Nacional de Desarrollo 2002-2006. Bogotá: Departamento Nacional de Planeación.

DNP (1998). Plan Nacional de Desarrollo 1998-2002. Bogotá: Departamento Nacional de Planeación.

DNP (1994). Plan Nacional de Desarrollo 1994-1998. Bogotá: Departamento Nacional de Planeación.

DNP (1990). Plan Nacional de Desarrollo 1990-1994. Bogotá: Departamento Nacional de Planeación.

Dwyer, L. y Kim, C. (2003a). "Destination Competitiveness and Bilateral Tourism Flows Between Australia and Korea". The Journal of Tourism Studies, 14(2), 55-67. DOI: 10.1016/ S0278-4319(99)00034-1

Dwyer, L. y Kim, C. (2003b). "Destination competitiveness: determinants and indicators". Current Issues in Tourism, 6(5), 369-414. DOI: 10.1080/13683500308667962

Fayós-Solá, E. (2004). “Política turística en la era de la globalización”. Mediterráneo Económico, 5, 215-235.

Guerrero, J.P. (1995). "La evaluación de políticas públicas: enfoques teóricos y realidades en nueve países desarrollados". Gestión y Política Pública, 4(1), 47-115.

Hall, C.M. (2008). Tourism planning: Policies, processes and relationships. Harlow: Prentice Hall.

Hall, C. M., Jenkins, J. y Kearsley, G. (1997). Tourism planning and policy in Australia and New Zealand: Cases, issues and practice. Sydney: McGraw Hill.

Hall, C.M. y Jenkins, J.M. (1995). Tourism and Public Policy. London: Routledge.

Hall, C.M. (1994). Tourism and politics: Policy, power and place. Chichester: Wiley.

Hall, C. M. (1989). The political power of economic ideas: Keynesianism across nations. Princeton: Princeton University Press. 
Hassan, S. (2000). "Determinants of market competitiveness in an environmentally sustainable tourism industry". Journal of Travel Research, 38, 239-245. DOI: 10.1177/004728750003800305

Izard, M. (2010). Gestión pública del Turismo. Barcelona: Editorial UOC.

Matthews, H.G. y Richter, L.K. (1991). “Political science and tourism”. Annals of Tourism Research, 18(1), 120-135. DOI: 10.1016/0160-7383(91)90043-B.

Meny, I. y Thoenig, J.C. (1992). Las políticas públicas. Barcelona: Ariel.

MinCIT - Ministerio de Comercio, Industria y Turismo - (2015). Informe turismo, marzo 2015. Bogotá: Ministerio de Comercio, Industria y Turismo de Colombia. En línea:

$<$ http://www.mincit.gov.co/publicaciones.php?id=16590> (consulta: 8 de junio 2015).

MinCIT - Ministerio de Comercio, Industria y Turismo - (2014). Plan Sectorial de Turismo 2014-2018. Bogotá: Ministerio de Comercio, Industria y Turismo de Colombia.

MinCIT - Ministerio de Comercio, Industria y Turismo - (2011). Plan Sectorial de Turismo 2011-2014. Bogotá: Ministerio de Comercio, Industria y Turismo de Colombia.

MinCIT - Ministerio de Comercio, Industria y Turismo - (2008). Plan Sectorial de Turismo 2008-2010. Bogotá: Ministerio de Comercio, Industria y Turismo de Colombia.

MinCIT - Ministerio de Comercio, Industria y Turismo - (2003). Plan Sectorial de Turismo 2003-2006. Bogotá: Ministerio de Comercio, Industria y Turismo de Colombia.

Ministerio de Transporte (2014). Transporte en cifras, estadísticas 2013. Bogotá: Ministerio de Transporte de Colombia, Bogotá.

Molina, R.; Ochoa, R. y Gil, A.M. (2014). "Public policies and tourism marketing. An analysis of the competitiveness on tourism in Morelia, Mexico and Alcala de Henares, Spain”. Procedia-Social and Behavioral Sciences, 148, 146-152. DOI: 10.1016/j.sbspro.2014.07.028

Monfort, V.M. (2000). “La política turística: una aproximación”. Cuadernos de Turismo, 6, 7-27.

Moreno, M. (1981). “Algunos aspectos históricos de la hotelería en Colombia”. Boletín de la Sociedad Geográfica de Colombia, 35(166), 1-8.

Peña, D. (2009). "Los instrumentos legales de la política turística como base para un turismo responsable y sostenible en Latinoamérica: Análisis para Colombia, Perú, México y Honduras”. Revista de Análisis Turístico, 8, 33-43.

Porter, M. (1990). “¿Dónde Radica la Ventaja Competitiva de las Naciones?”. Harvard-Deusto Business Review, septiembre/diciembre, 3-26.

Presidencia de la República (2015). Diálogos para la terminación del conflicto armado. Presidencia de la República de Colombia. Bogotá, Colombia. En Linea: <http://wsp.presidencia.gov.co/portal/Especiales/Paginas/ Especial-Terminacion-Conflicto.aspx> (consulta: 8 de junio 2015).

Quintero, P.A. (2009). Desarrollo y competitividad del turismo en Colombia: Balance de las políticas y la gestión turística. Barcelona: Universitat de Barcelona.

Restrepo, O. (2014). "Análisis de la política pública turística para la transformación productiva del sector en Colombia 2004-2014”. Anais Brasileiros de Estudios Turísticos, 4(3), 18-34.

Ritchie, J.R.B.; Crouch, G.I. y Hudson, S. (2001). "Developing operational measures for the components of a destination competitiveness/sustainability model: consumer versus managerial perspectives". En Mazanec, J.A.; Crouch, J.R.B. \& Wooside, A.G. (Eds.) Consumer Psychology of Tourism, Hospitality and Leisure, Vol. 2, $1-17$.

Richter, L. K. (1983). "Tourism politics and political science: A case of not so benign neglect". Annals of Tourism Research, 10(3), 313-335. DOI: 10.1016/0160-7383(83)90060-9

Rodríguez, G. y Martínez, F. (2009). Nuevos retos para el turismo. La Coruña: Editorial Netbiblo.

Roth Deubel, A.N. (2002). Políticas públicas. Formulación, implementación y evaluación. Bogotá: Ed. Aurora.

Scott, N. (2011). Tourism policy: A strategic review. Oxford: Goodfellow Publishers.

Toro, G. (2003). “La política pública de turismo en Colombia”. Turismo y Sociedad, 2(2), 9-15.

Torres, E. (2004). “Del turismo en la política económica a la política económica del turismo”. Quaderns de Política Econòmica, 7, 49-71.

UNWTO (2014). Compendium of Tourism Statistics, 2014 Edition. Madrid: World Tourism Organization.

UNWTO (2009). Colombia, back on the map of world tourism. Madrid: World Tourism Organization.

UNWTO \& ETC (2009). Handbook on Tourism Destinations Branding. Madrid: World Tourism Organization and European Travel Commission.

Velasco, M. (2011). “La política turística. Una arena de acción autonómica”. Cuadernos de Turismo, 27, $953-969$.

Velasco, M. (2004). La política turística. Gobierno y administraciones turísticas en España (1952-2004). Valencia: Fundación Cañada Blanch.

Velásquez, R. (2009). “Hacia una nueva definición de política pública”. Desafíos, 20, 149-187. 
WEF (2015). The Travel \& Tourism Competitiveness Report 2015. Geneva: World Economic Forum.

WEF (2013). The Travel \& Tourism Competitiveness Report 2013. Geneva: World Economic Forum.

WEF (2007). The Travel \& Tourism Competitiveness Report 2007. Geneva: World Economic Forum.

WEF (2006). The Global Competitiveness. Report 2006-2007. Geneva: World Economic Forum.

Whitford, M.M. (2005). An interpretive analysis of event policy: South East Queensland regional organisation of councils 1974-2004. Ph.D. Thesis, The University of Queensland.

Zúñiga, A. y Castillo, M. (2012). "Turismo en Colombia: resultados del sector (2007-2010)". Magazine Empresarial, $8(15), 67-73$.

Zúñiga, A.; Castillo, M; Miki, A. (2012). "Análisis de la producción de investigación científica internacional sobre turismo en Colombia y Brasil y el desarrollo turístico actual de los países”. Turismo em Análise, 23(2), $240-264$. 